Bull. Austral. Math. Soc.

Vol. 43 (1991) [407-422]

\title{
TOPOLOGICAL DIMENSIONS OF GLOBAL ATTRACTORS FOR SEMILINEAR PDE'S WITH DELAYS
}

\author{
JOSEPH W.-H. So AND JiANHONG WU
}

\begin{abstract}
An estimate is obtained on the Hausdorff and fractal dimensions of global attractors of semilinear partial differential equations with delay: $\dot{x}(t)=A x(t)+f\left(x_{t}\right)$. The method employed is to associate such an equation with a nonlinear semigroup on a product space and then appeal to the upper estimate due to Constantin, Foias and Teman on topological dimensions of global attractors for general nonlinear dynamical systems.
\end{abstract}

\section{INTRODUCTION.}

Recent studies of nonlinear dynamical systems show that many infinite-dimensional evolution equations have global attractors with finite Hausdorff and fractal dimensions. In particular, this fact has been demonstrated by Babin and Vishik $[1,2]$ and Constantin, Foias and Teman [3] for Navier-Stokes equations, Koppel and Ruelle [8], Marion $[11,12]$ for reaction diffusion equations and Mallet-Paret [10] for (ordinary) functional differential equations.

The purpose of this paper is to extend these results to a class of semilinear partial differential equations with delay of the form:

$$
\begin{aligned}
& \dot{x}(t)=A x(t)+f\left(x_{t}\right), \quad t \geqslant 0 \\
& x(0)=h \in H, \quad x_{0}=\phi \in L^{2}([-r, 0] ; H)
\end{aligned}
$$

where $H$ is a Hilbert space, $r>0$ is a given constant, $A: D(A) \rightarrow H$ generates a strongly continuous semigroup on $H$ and $f: L^{2}([-r, 0] ; H) \rightarrow B$ is everywhere defined and Lipschitz continuous. As is well known, a major difficulty in obtaining such an extension is caused by the fact that the variational equation

$$
\dot{u}(t)=A u(t)+D f\left(x_{t}\right) u_{t}, \quad t>0
$$

\footnotetext{
Received 6th June 1990.
}

The first author's research was partially supported by the Natural Sciences and Engineering Research Council of Canada, grant number NSERC OGP36475. The second author's research was partially supported by a Gordin Kaplan Postdoctoral Fellowship at the University of Alberta.

Copyright Clearance Centre, Inc. Serial-fee code: 0004-9729/91 \$A2.00+0.00. 
of (FDE) about a given solution $x($.$) is not formulated on the state space X=$ $L^{2}([-r, 0] ; H) \times H$ so that the upper estimate due to Constantin, Foias and Teman [3] cannot be applied to our case directly.

Our approach is to extend the generator $A$ (in $H$ ) to $\bar{A}$ on the state space $X$ in a natural way (Proposition 3.2) so that the variational equation (VE) can be reformulated as an evolution system on $X$ of the form

$$
\dot{U}(t)=\bar{A} U(t)+\left\{0, D f\left(x_{t}\right) P_{1} U(t)\right\}
$$

where $P_{1}$ denotes the projection of $X$ onto $L^{2}([-r, 0] ; H)$. This is motivated by the papers by Travis and Webb $[15,16]$ and Webb $[17,18]$ where they constructed a generator for a nonlinear semigroup and associated such a semigroup with the solutions of (FDE) by using results from the general nonlinear semigroup theory.

The rest of the paper is organised as follows. In Section 2, we collect some general results from the theory of nonlinear semigroups, dissipative dynamical systems and topological dimensions of global attractors. In Section 3, we apply the general results of Webb $[17,18]$ to (FDE) and obtain a strongly continuous semigroup $\{T(t)\}_{t \geqslant 0}$ on the product space $X$ (Theorem 3.1). Then from the generation formula of semigroups due to Crandall and Liggett [4], we establish a variation-of-constants formula for variational equations of (FDE) (Proposition 3.4) which is then applied to yield the relation between (EVE) and (VE) (Proposition 3.6). Finally, we show that the semigroup $\{T(t)\}_{t \geqslant 0}$ is uniformly differentiable with respect to the global attractor and that, because $D(\bar{A})$ is dense in $X$, the upper estimate due to Constantin, Foias and Teman [3] can then be applied to obtain our main result (Theorem 3.10) on the topological dimensions of global attractors for (FDE).

The verification of the hypotheses in Theorem 3.10 for reaction-diffusion systems with delays is a non-trivial task and will be reported in a future paper.

\section{Semigroups, Evolution Equations and Global attractors.}

In this section, we set forth some necessary preliminaries from the theory of nonlinear semigroups, dissipative dynamical systems and topological dimensions of global attractors.

Throughout this section, $X$ is a Hilbert space with inner product $(., .)_{X}$ and norm |.|x

A (nonlinear) strongly continuous semigroup $\{T(t)\}_{t \geqslant 0}$ on $X$ is a family of (everywhere defined) continuous mappings $T(t): X \rightarrow X, t \geqslant 0$, satisfying the following properties:

(i) $T(0)=I$ (identity), 
(ii) $T(t+s)=T(t) T(s)$ for all $t, s \geqslant 0$, and

(iii) for each $x \in X, T() x:.[0, \infty) \rightarrow X$

is continuous.

A strongly continuous semigroup has a densely defined generator. The following "generation" theorem is due to Crandall and Liggett [4]. (See also Kato [7].)

ThEOREM 2.1. Suppose that

(i) $A: D(A) \subset X \rightarrow X$, where $D(A)$ is a dense subset of $X$,

(ii) for some $\gamma \in \mathrm{R}, \gamma I-A: D(A) \rightarrow X$ is monotone (accretive), and

(iii) $R(I-\lambda A)=X$ for all $\lambda>0$ sufficiently small.

Then $T(t) x \equiv \lim _{n \rightarrow \infty}[I-t / n A]^{-n} x$ exists for every $x \in X$ and $t \geqslant 0$. Moreover, $\{T(t)\}_{t \geqslant 0}$ is a strongly continuous semigroup on $X$ and

$$
|T(t) x-T(t) y|_{X} \leqslant e^{\gamma t}|x-y|_{X}, \quad \text { for all } \quad x, y \in X, \quad t \geqslant 0 .
$$

$A$ is called a generator for the semigroup $\{T(t)\}_{t \geqslant 0}$.

For the rest of this section, we let $\{T(t)\}_{t \geqslant 0}$ be a (fixed) strongly continuous semigroup on $X$. The following notions leading to the concept of a global attractor and its existence can be found in Hale [6]. We include them here for the sake of convenience.

A subset $Y \subset X$ is said to be positively invariant (respectively invariant) if $T(t) Y \subset Y$ (respectively $T(t) Y=Y$ ) for all $t \geqslant 0$. A compact invariant set is said to be a maximal compact invariant set if it contains all compact invariant sets. A global attractor $\Omega$ is a maximal compact invariant set such that $\delta(T(t) B, \Omega) \rightarrow 0$ as $t \rightarrow \infty$, for every bounded set $B \subset X$. Here, $\delta\left(B_{0}, B_{1}\right)$ denotes semi-distance of two sets $B_{0}, B_{1} \subset X$, that is $\delta\left(B_{0}, B_{1}\right):=\sup _{x \in B_{0}} \inf _{y \in B_{1}}|x-y|_{x}$.

Obviously, $\Omega$ is unique and the dynamics on a global attractor include all the possible asymptotic behaviour (that is, as $t \rightarrow \infty$ ) of the given semigroup $\{T(t)\}_{t \geqslant 0}$. To guarantee the existence of such an attractor, we introduce the following concepts. The semigroup $\{T(t)\}_{t \geqslant 0}$ is said to be bounded dissipative if, there is a bounded set $B_{0} \subset X$ such that for every bounded set $B \subset X$ there exists $t\left(B_{0}\right)>0$ such that $T(t) B \subset B_{0}$ for all $t \geqslant t\left(B_{0}\right)$. The semigroup $\{T(t)\}_{t \geqslant 0}$ is asymptotically smooth if for any non-empty positively invariant set $B \subset X$ there exists a compact set $J \subset B$ such that $\delta(T(t) B, J) \rightarrow 0$ as $t \rightarrow \infty$. An example of an asymptotically smooth semigroup is one which is uniformly compact, that is, for every bounded set $B$, there exists $t_{0} \geqslant 0$ such that $\underset{t \geqslant t_{0}}{\cup} T(t) B$ has compact closure.

The following existence result for global attractor can be found in Hale [6]. 
THEOREM 2.2. If a strongly continuous semigroup $\{T(t)\}_{t \geqslant 0}$ on $X$ is asymptotically smooth and bounded dissipative, then $\{T(t)\}_{t \geqslant 0}$ possesses a global attractor $\Omega$.

Next, we will recall the concept of Hausdorff and fractal dimensions of a compact set $\Omega \subset X$. More details can be found in Teman [14], Federler [5] and Mandelbrot [9].

For any $d>0$, let $\mu_{H}(\Omega, d)=\lim _{e \rightarrow 0} \mu_{H}(\Omega, d, \varepsilon)$ denote the $d$-dimensional Hausdroff measure of the set $\Omega \subset X$, where $\mu_{H}(\Omega, d, \varepsilon)=\inf \sum_{i} r_{i}^{d}$ and the infimum is taken over all coverings of $\Omega$ by balls of radius $r_{i} \leqslant \varepsilon$. It can be shown that there exists $d_{H}(\Omega) \in[0,+\infty]$ such that $\mu_{H}(\Omega, d)=0$ for $d>d_{H}(\Omega)$ and $=\infty$ for $d<d_{H}(\Omega)$. $d_{H}(\Omega)$ is called the Hausdroff dimension of $\Omega$.

The fractal dimension (or capacity) of $\Omega$ is defined as

$$
d_{F}(\Omega)=\inf \left\{d>0: \mu_{F}(\Omega, d)=0\right\}
$$

where $\mu_{F}(\Omega, d)=\limsup _{\varepsilon \rightarrow 0} \varepsilon^{d} n_{F}(\Omega, \varepsilon)$ and $n_{F}(\Omega, \varepsilon)$ is the minimum number of balls of radius $\leqslant \varepsilon$ which is necessary to cover $\Omega$.

In order to use linearisation to estimate the Hausdorff and fractal dimension of the global attractor $\Omega$ of a strongly continuous semigroup $\{T(t)\}_{t \geqslant 0}$ later on, we need the following notions. $\{T(t)\}_{t \geqslant 0}$ is said to be uniformly differentiable in $\Omega$ if, for every $x \in \Omega$ and $t \geqslant 0$ there exists a bounded linear operator $L(t, x): X \rightarrow X$ such that

$$
\sup _{x, y \in \Omega, 0<|x-y| \leqslant e} \frac{|T(t) y-T(t) x-L(t, x)(y-x)| x}{|y-x| x} \rightarrow 0 \quad \text { as } \varepsilon \rightarrow 0,
$$

and

$$
\sup _{x \in \Omega}|L(t, x)|_{B L(X ; X)}<+\infty
$$

where $B L(X, X)$ is the Banach space of bounded linear operators on $X$ with the usual operator norm $|\cdot|_{B L(X, X)}$.

Let $\{T(t)\}_{t \geqslant 0}$ be uniformly differentiable in $\Omega, x \in \Omega$ and let $N$ be a given positive integer. We denote by $\omega_{N}(L(t, x))$ the norm of the exterior product $\wedge^{N} L(t, x)$ : $\wedge^{N} X \rightarrow \wedge^{N} X$, that is,

$$
\omega_{N}(L(t, x))=\sup _{\xi^{1}, \ldots, \xi^{N} \in X,\left|\xi^{i}\right| \leqslant 1}\left|L(t, x) \xi^{1} \wedge \cdots \wedge L(t, x) \xi^{N}\right|_{\wedge^{N} X}
$$

Set

$$
\bar{\omega}_{N}(t)=\sup _{x \in \Omega} \omega_{N}(L(t, x)), \quad t \geqslant 0
$$


and

$$
\pi_{N}=\lim _{t \rightarrow \infty} \bar{\omega}_{N}(t)^{1 / t}
$$

The uniform Lyapunov numbers are defined as follows:

$$
\mu_{1}=\ln \pi_{1}
$$

and

$$
\mu_{N}=\ln \pi_{N}-\ln \pi_{N-1}, \quad \text { for } \quad N \geqslant 2 .
$$

The following upper estimate on the Hausdorff and fractal dimensions of a global attractor was proved in Constantin, Foias and Teman [3].

Theorem 2.3. Assume that the hypotheses in Theorem 2.2 hold and the strongly continuous semigroup $\{T(t)\}_{t \geqslant 0}$ is uniformly differentiable in the global attractor $\Omega \subset X$. If there exists an integer $N \geqslant 1$ such that

$$
\begin{gathered}
\mu_{1}+\ldots \mu_{N}<0 \\
d_{H}(\Omega) \leqslant N
\end{gathered}
$$

then

and

$$
d_{F}(\Omega) \leqslant N \max _{1 \leqslant l \leqslant N-1}\left\{1+\frac{\left(\mu_{1}+\cdots+\mu_{l}\right)_{+}}{\left|\mu_{1}+\cdots+\mu_{N}\right|}\right\} .
$$

In order to investigate the uniform differentiability of a semigroup, we need to consider the following semilinear initial value problem:

$$
\begin{cases}d u(t) / d t & =A u(t)+f(t, u(t)), \quad t>0 \\ u(0) & =u_{0} \in X,\end{cases}
$$

where $A: D(A) \subset X \rightarrow X$ generates a strongly continuous semigroup of bounded linear operators $T(t): X \rightarrow X, t \geqslant 0$ and the mapping $f:[0, \infty) \times X \rightarrow X$ satisfies the following properties: $f(., x):[0, \infty) \rightarrow X$ is continuous for each fixed $x \in X$, and for any constant $t_{0}>0, f(t, x)$ is uniformly Lipschitz continuous in $x \in X$ for all $t \in\left[0, t_{0}\right]$.

A strong solution of $(2.10)$ is a continuous function $u:[0, \infty) \rightarrow X$ which is (i) continuously differentiable on $(0, \infty)$, (ii) $u(t) \in D(A)$ for $t>0$ and (iii) (2.10) is satisfied on $(0, \infty)$. A mild solution is a continuous function $u:[0, \infty) \rightarrow X$ satisfying the integral equation

$$
u(t)=T(t) u_{0}+\int_{0}^{t} T(t-s) f(s, u(s)) d s, \quad t \geqslant 0 .
$$

The following existence and regularity result can be found in Pazy [13].

Theorem 2.4. For every $u_{0} \in X$, the initial value problem (2.10) has a unique mild solution $u:[0, \infty) \rightarrow X$ and the mapping $u_{0} \mapsto u$ is Lipschitz continuous from $X$ to $C\left(\left[0, t_{0}\right] ; X\right)$ for each $t_{0}>0$. Moreover, if $f:[0, \infty) \times X \rightarrow X$ is continuously differentiable, then the mild solution of (2.10) with $u_{0} \in D(A)$ is a strong solution. 


\section{Dimensions of Global attractors for Abstract fDEs.}

We now consider the following initial value problem of abstract semilinear functional differential equations (FDEs)

$$
\left\{\begin{array}{l}
\dot{x}(t)=A x(t)+f\left(x_{t}\right), \quad t>0 \\
x(0)=h \in H, \quad x_{0}=\phi \in L^{2}([-r, 0] ; H)
\end{array}\right.
$$

where $H$ is a Hilbert space with an inner product $(.,)_{H}$ and norm $|\cdot|_{H}, r>0$ is fixed, $x_{t}$ is the standard notation meaning that if $x:(-r, \infty) \rightarrow H$ and $t \geqslant 0$, then $x_{t}:[-r, 0] \rightarrow H$ is defined as $x_{t}(\theta)=x(t+\theta)$ for almost all $\theta \in[-r, 0] . f:$ $L^{2}([-r, 0] ; H) \rightarrow H$ is everywhere defined and Lipschitz continuous with Lipschitz constant $\beta$. $A: D(A) \subset H \rightarrow H$ is a densely defined linear operator such that $\alpha I-A$ is monotone in $H$ for some $\alpha \in \mathbf{R}$ and that $R(I-\lambda A)=A$ for sufficiently small $\lambda>0$.

Under the above assumptions on $A$, by Theorem 2.1 due to Crandall and Liggett [4], $A$ generates a strongly continuous semigroup $\{S(t)\}_{t \geqslant 0}$ of bounded linear operators defined by

$$
S(t) h=\lim _{n \rightarrow \infty}\left[I-\frac{t}{n} A\right]^{-n} h, \quad t \geqslant 0, \quad h \in H .
$$

Moreover, $|S(t)|_{B L(H ; H)} \leqslant e^{\alpha t}, \quad t \geqslant 0$.

Following Webb $[17,18]$, we will treat the initial value problem (FDE) in the Hilbert space $X=L^{2}([-r, 0] ; H) \times H$ with inner product

$$
(\{\phi, h\},\{\psi, k\})_{X}=\int_{-r}^{0}(\phi(\theta), \psi(\theta))_{H} d \theta+(h, k)_{H} \quad \text { for } \quad\{\phi, h\},\{\psi, k\} \in X
$$

and norm

$$
|\{\phi, h\}|_{X}=(\{\phi, h\},\{\phi, h\})_{X}^{1 / 2} \quad \text { for } \quad\{\phi, h\} \in X .
$$

Define $B: D(B) \subset X \rightarrow X$ by

$$
\begin{gathered}
D(B)=\{\{\phi, h\} \in X: \phi:[-r, 0] \rightarrow H \text { is absolutely continuous, } \\
\left.\dot{\phi} \in L^{2}([-r, 0] ; H) \text { and } h=\phi(0) \in D(A)\right\}, \\
B\{\phi, h\}=\{\dot{\phi}, A h+f(\phi)\}, \quad \text { for }\{\phi, h\} \in D(B) .
\end{gathered}
$$

The following result was proved in Webb [17]. 
THEOREM 3.1. Under the above hypotheses, we have

(i) $B$ is densely defined, $\gamma I-B$ is monotone in $X$ with $\gamma=\max$ $\{0,1 / 2+\alpha\}+\beta$, and $R(I-\lambda B)=X$ for sufficiently small $\lambda>0$.

(ii) $B$ generates a strongly continuous semigroup $\{T(t)\}_{t \geqslant 0}$ on $X$ defined by

$$
T(t)\{\phi, h\}=\lim _{n \rightarrow \infty}\left[I-\frac{t}{n} B\right]^{-n}\{\phi, h\}, \quad\{\phi, h\} \in X, \quad t \geqslant 0 .
$$

(iii) $|T(t)\{\phi, h\}-T(t)\{\psi, k\}| x \leqslant e^{\gamma t}|\{\phi, h\}-\{\psi, k\}| x$, for $\{\phi, h\},\{\psi, k\} \in X$ and $t \geqslant 0$.

(iv) Let $P_{1}$ and $P_{2}$ denote the projections of $X$ onto $L^{2}([-r, 0] ; H)$ and $H$ respectively and for every $\{\phi, h\} \in X$, define $x^{\phi, h}:[-r, \infty) \rightarrow H$ by

$$
x^{\phi, h}(t)= \begin{cases}\phi(t) & -r \leqslant t<0, \\ P_{2} T(t)\{\phi, h\} & t \geqslant 0 .\end{cases}
$$

Then $x_{i}^{\phi, h}=P_{1} T(t)\{\phi, h\}$ for $t \geqslant 0$.

(v) If $\{\phi, h\} \in D(B)$, then

$$
\dot{x}^{\phi, h}(t)=A x^{\phi, h}(t)+f\left(x_{t}^{\phi, h}\right), \quad \text { for almost everywhere } t \geqslant 0 .
$$

REMARK: As a consequence of (iv), we have

$$
P_{1} T(t)\{\phi, h\}(\theta)=\phi(t+\theta) \quad \text { for } t \geqslant 0, t+\theta<0 .
$$

To reformulate (FDE) as an abstract ordinary differential equation we introduce the following operator $\bar{A}$ defined in $X$ :

$$
\begin{gathered}
D(\bar{A})=\{\{\phi, h\} \in X: \phi \text { is absolutely continuous on }[-r, 0], \\
\left.\dot{\phi} \in L^{2}([-r, 0] ; H) \text { and } h=\phi(0) \in D(A)\right\} \\
\bar{A}\{\phi, h\}=\{\dot{\phi}, A h\}, \quad\{\phi, h\} \in D(\bar{A}) .
\end{gathered}
$$

By Theorem 3.1, it follows immediately that $\bar{A}$ generates a strongly continuous semigroup $\{\bar{S}(t)\}_{t \geqslant 0}$ of bounded linear operators on $X$ by the formula:

$$
\bar{S}(t)\{\phi, h\}=\lim _{n \rightarrow \infty}\left[I-\frac{t}{n} \bar{A}\right]^{-n}\{\phi, h\}, \quad\{\phi, h\} \in X, \quad t \geqslant 0 .
$$

Moreover, we have

$$
|\bar{S}(t)|_{B L(X, X)} \leqslant e^{\max \{0,1 / 2+\alpha\} t}, \quad t \geqslant 0 .
$$

The following result shows that $\{S(t)\}_{t \geqslant 0}$ is exactly the projection onto $H$ of the semigroup $\{\bar{S}(t)\}_{t \geqslant 0}$. 
Proposition 3.2. $S(t) h=P_{2} \bar{S}(t)\{\phi, h\}$ for all $\{\phi, h\} \in X$.

Proof: It is easily seen that $P_{2}[I-t / n \bar{A}]^{n}\{\phi, h\}=[I-t / n A]^{-n} h$ for all $\{\phi, h\} \in$ $X, t \geqslant 0$ and positive integer $n$. Therefore by (3.1) and (3.3), we get $P_{2} \bar{S}(t)\{\phi, h\}$ $=P_{2} \lim _{n \rightarrow \infty}[I-t / n \bar{A}]^{-n}\{\phi, h\}=\lim _{n \rightarrow \infty} P_{2}[I-t / n \bar{A}]^{-n}\{\phi, h\}=\lim _{n \rightarrow \infty}[I-t / n A]^{-n} h$ $=S(t) h$.

Throughout the remainder of this section, we assume that $\{T(t)\}_{t \geqslant 0}$ satisfies the following conditions.

(H1) $\{T(t)\}_{t \geqslant 0}$ is bounded dissipative,

(H2) $\{T(t)\}_{t \geqslant 0}$ is uniformly compact, and

(H3) $T(t) X \subset D(B)$ for all $t \geqslant 2 r$.

PROPOSITION 3.3. The semigroup $\{T(t)\}_{t \geqslant 0}$ has a global attractor $\Omega$ and $\Omega \subset D(B)$.

Proof: The existence of $\Omega$ is an immediate consequence of Theorem 2.2. The conclusion that $\Omega \subset D(B)$ follows from (H3) and the invariance of $\Omega$.

To study the differentiability of $\{T(t)\}_{t \geqslant 0}$ with respect to initial data, we consider the variational equation:

$$
\begin{cases}d U(t) / d t & =\bar{A} U(t)+\left\{0, D f\left(x_{t}^{\phi, h}\right) P_{1} U(t)\right\}, \quad t>0 \\ U(0) & =\{\psi, k\} \in X\end{cases}
$$

where $\{\phi, h\} \in \Omega$ is given.

Proposition 3.4. If $f: L^{2}([-r, 0] ; H)$ is twice continuously differentiable $\left(C^{2}\right)$, then for each $\{\phi, h\} \in \Omega$ and $\{\psi, k\} \in X$, there exists a continuous function $U=U^{(\phi, h, \psi, k)}:[0, \infty) \rightarrow X$ such that

$$
U(t)=\bar{S}(t)\{\psi, k\}+\int_{0}^{t} \bar{S}(t-s)\left\{0, D f\left(x^{\phi, h}\right) P_{1} U(s)\right\} d s, \quad t \geqslant 0
$$

Moreover, if $\{\psi, k\} \in D(\bar{A})$, then $U(t)$ is a strong solution of (3.5).

Proof: Let $L_{1}=\sup _{\Phi \in P_{1} \Omega}|D f(\Phi)|$, where

$$
|D f(\Phi)|=\sup _{\Psi \in L^{2}([-r, 0] ; H),|\Psi|_{L^{2}([-r, 0] ; H)} \leqslant 1}|D f(\Phi) \Psi|_{H} .
$$

Since $f$ is $C^{1}$ and $P_{1} \Omega$ is compact, $L_{1}<\infty$. Given any $\{\phi, h\} \in \Omega$, define $F_{\phi, h}$ : $[0, \infty) \times X \rightarrow X$ by

$$
F_{\phi, h}(t,\{\psi, k\})=\left\{0, D f\left(x_{t}^{\phi, h}\right) \psi\right\}, \quad t \geqslant 0, \quad\{\psi, k\} \in X .
$$


Since $\{\phi, h\} \in \Omega \subset D(B), x^{\phi, h}:[0, \infty) \rightarrow L^{2}([-r, 0] ; H)$ is $C^{1}$. Therefore, by the assumption that $f: L^{2}([-r, 0] ; H) \rightarrow H$ is $C^{2}$, it follows that $F_{\phi, h}$ is $C^{1}$. Moreover, the invariance of $\Omega$ for the semigroup $\{T(t)\}_{t \geqslant 0}$ guarantees that $x_{t}^{\phi, h} \in P_{1} \Omega$ and thus $\left|D f\left(x_{t}^{\phi, h}\right)\right| \leqslant L_{1}<\infty$, for all $t \geqslant 0$. This implies that $F_{\phi, h}(t,$.$) is Lipschitz$ continuous on $X$, uniformly for all $t \in[0, \infty)$. Therefore the conclusion follows from Theorem 2.4 .

From now on, we assume that $f$ is $C^{2}$. The following result shows that $P_{1} U^{\phi, h, \psi, k}(t)$ is the segment of $P_{2} U^{\phi, h, \psi, k}(t)$ on $[t-r, t]$.

Proposition 3.5. Let $u^{\phi, h, \psi, k}(t)=P_{2} U^{\phi, h, \psi, k}(t)$, where $\{\phi, h\} \in \Omega$ and $\{\psi, k\} \in X$. Then $u_{t}^{\phi, h, \psi, k}=P_{1} U^{\phi, h, \psi, k}(t)$.

Proof: To simplify notation, we set $U(t)=U^{\phi, h, \psi, k}(t)$ and $u(t)=u^{\phi, h, \psi, k}(t)$. Applying (iv) of Theorem 3.1 to the semigroup $\{\bar{S}(t)\}_{t \geqslant 0}$, we get $P_{1} \bar{S}(t)\{\psi, k\}(\theta)=$ $P_{2} \bar{S}(t+\theta)\{\psi, k\}$, for $t \geqslant 0, \theta \in[-r, 0]$ and $t+\theta \geqslant 0$. Applying $P_{2}$ on both sides of $(3,6)$ at $t+\theta$, we get

$$
\begin{aligned}
P_{2} U(t+\theta) & =P_{2} \bar{S}(t+\theta)\{\psi, k\}+P_{2} \int_{0}^{t+\theta} \bar{S}(t+\theta-s)\left\{0, D f\left(x_{a}^{\phi, h}\right) P_{1} U(s)\right\} d s \\
& =P_{1} \bar{S}(t)\{\psi, k\}(\theta)+\int_{0}^{t+\theta} P_{1} \bar{S}(t-s)\left\{0, D f\left(x_{\bullet}^{\phi, h}\right) P_{1} U(s)\right\}(\theta) d s \\
& =P_{1} \bar{S}(t)\{\psi, k\}(\theta)+P_{1} \int_{0}^{t} \bar{S}(t-s)\left\{0, D f\left(x_{\bullet}^{\phi, h}\right) P_{1} U(s)\right\}(\theta) d s \\
& =P_{1} U(t)(\theta)
\end{aligned}
$$

where the equality

$\int_{0}^{t+\theta} P_{1} \bar{S}(t-s)\left\{0, D f\left(x_{s}^{\phi, h}\right) P_{1} U(s)\right\}(\theta) d s=P_{1} \int_{0}^{t} \bar{S}(t-s)\left\{0, D f\left(x_{s}^{\phi, h}\right) P_{1} U(s)\right\}(\theta) d s$

holds because by the Remark following Theorem 3.1, $P_{1} \bar{S}(t-s)\left\{0, D f\left(x_{\theta}^{\phi, h}\right) P_{1} U(s)\right\}(\theta)$ $=0$, whenever $t+\theta-s \leqslant 0$. The conclusion then follows from the definition of $P_{2}$ and $u_{t}$.

Proposition 3.6. If $\{\phi, h\} \in \Omega$ and $\{\psi, k\} \in D(A)$ then $u^{\psi, h, \psi, k}:[0, \infty) \rightarrow$ $\boldsymbol{H}$ is differentiable and

$$
\dot{u}^{\phi, h, \psi, k}(t)=A u^{\phi, h, \psi, k}+D f\left(x_{t}^{\phi, h}\right) u_{t}^{\phi, h, \psi, k}, \quad t \geqslant 0 .
$$

Proof: Since $\{\phi, h\} \in \Omega$ and $\{\psi, k\} \in D(A)$, by Proposition 3.4, $U^{\phi, h, \psi, k}$ is a strong solution of (3.5). Therefore $u^{\phi, h, \psi, k}=P_{2} U^{\phi, h, \psi, k}:[0, \infty) \rightarrow H$ is differentiable. Applying $P_{2}$ on both sides of (3.5) and using Proposition 3.5, we obtain (3.7). 
Proposition 3.7. For any constant $t_{0}>0$, there exists a constant $L>0$ such that

$\left|x_{t}^{\phi, h}-x_{t}^{\psi, k}\right|_{x} \leqslant L|\{\phi, h\}-\{\psi, k\}|_{x}, \quad$ for all $\quad\{\phi, h\},\{\psi, k\} \in \Omega, \quad t \in\left[0, t_{0}\right]$

Proof: To simplify notation, we set $x(t)=x^{\phi, h}(t)$ and $y(t)=x^{\psi, k}(t)$. Since $\{\phi, h\},\{\psi, k\} \in D(B)$, by Proposition 3.1, we have

$$
\begin{array}{ll}
\dot{x}(t)=A x(t)+f\left(x_{t}\right), & t \in\left[0, t_{0}\right] \quad \text { and } \\
\dot{y}(t)=A y(t)+f\left(y_{t}\right), & t \in\left[0, t_{0}\right],
\end{array}
$$

from which it follows that

$$
\begin{array}{ll}
x(t)=S(t) h+\int_{0}^{t} S(t-s) f\left(x_{s}\right) d s, & t \in\left[0, t_{0}\right] \\
y(t)=S(t) k+\int_{0}^{t} S(t-s) f\left(y_{2}\right) d s, & t \in\left[0, t_{0}\right] .
\end{array}
$$

Therefore

$$
x(t)-y(t)=S(t)(h-k)+\int_{0}^{t} S(t-s)\left[f\left(x_{s}\right)-f\left(y_{s}\right)\right] d s, \quad t \in\left[0, t_{0}\right] .
$$

Since $|S(t)|_{B L(H ; H)} \leqslant e^{\alpha t}$ for $t \in\left[0, t_{0}\right]$, we have

$$
\begin{aligned}
|x(t)-y(t)|_{H} & \leqslant e^{\alpha t}|h-k|_{H}+\int_{0}^{t} e^{\alpha(t-s)} \beta\left|x_{s}-y_{s}\right|_{L^{2}} d s \\
& \leqslant \max \left\{e^{\alpha t_{0}}, 1\right\}\left[|h-k|_{H}+\beta \int_{0}^{t}\left|x_{s}-y_{s}\right|_{L^{2}} d s\right]
\end{aligned}
$$

where for any $\rho \in L^{2}([-r, 0] ; H),|\rho|_{L^{2}}=\left(\int_{-r}^{0}|\rho(\theta)|_{H}^{2} d \theta\right)^{1 / 2}$. Thus by using the 
inequality $(a+b)^{2} \leqslant 2\left(a^{2}+b^{2}\right)(a, b \in \mathbf{R})$, we obtain

$$
\begin{aligned}
& \int_{-r}^{0}|x(t+\theta)-y(t+\theta)|_{H}^{2} d \theta \\
& =\int_{[t-r, t] \cap[-r, 0]}|x(\xi)-y(\xi)|_{H}^{2} d \xi+\int_{[t-r, t] \backslash[-r, 0]}|x(\xi)-y(\xi)|_{H}^{2} d \xi \\
& \leqslant \int_{[t-r, t] \cap[-r, 0]}|\phi(\xi)-\psi(\xi)|_{H}^{2} d \xi \\
& +2\left(\max \left\{e^{\alpha t_{0}}, 1\right\}\right)^{2} \int_{[t-r, t] \backslash[-r, 0]}\left[|h-k|_{H}^{2}+\beta^{2}\left(\int_{0}^{\xi}\left|x_{\bullet}-y_{\bullet}\right|_{L^{2}} d s\right)^{2}\right] d \xi \\
& \leqslant \int_{-r}^{0}|\phi(\xi)-\psi(\xi)|_{H}^{2} d \xi+2\left(\max \left\{e^{\alpha t_{0}}, 1\right\}\right)^{2} \int_{-r}^{0}\left[|h-k|_{H}^{2}+\beta^{2}\left(\int_{0}^{t}\left|x_{\triangleleft}-y_{\bullet}\right|_{L^{2}} d s\right)^{2}\right] d \xi \\
& \leqslant \max \left\{1,2 r\left(\max \left\{e^{\alpha t_{0}}, 1\right\}\right)^{2}\right\}|\{\phi, h\}-\{\psi, k\}|_{X}^{2} \\
& +2\left(\max \left\{e^{\alpha t_{0}}, 1\right\}\right)^{2} \beta^{2} r\left(\int_{0}^{t}\left|x_{\diamond}-y_{\bullet}\right|_{L^{2}} d s\right)^{2} .
\end{aligned}
$$

From the inequality $\sqrt{a^{2}+b^{2}} \leqslant|a|+|b|(a, b \in \mathbf{R})$, it follows that

$$
\left|x_{t}-y_{t}\right|_{L^{2}} \leqslant \sqrt{\max \left\{1, M_{0}\right\}}|\{\phi, h\}-\{\psi, k\}|_{X}+\sqrt{M_{0}} \beta \int_{0}^{t}\left|x_{s}-y_{\iota}\right|_{L^{2}} d s,
$$

where $M_{0}=2\left(\max \left\{e^{\alpha t_{0}}, 1\right\}\right)^{2} r$. By the well-known Gronwall inequality, we obtain

$$
\left.\left|x_{t}-y_{t}\right|_{L^{2}} \leqslant \sqrt{\max \left\{1, M_{0}\right.}\right\}|\{\phi, h\}-\{\psi, k\}|_{X} e^{\sqrt{M_{0}} \beta t} .
$$

Substituting (3.11) into (3.10), we get

$|x(t)-y(t)|_{H} \leqslant \max \left\{e^{\alpha t_{0}}, 1\right\}\left[|h-k|_{H}+\sqrt{\frac{\max \left\{1, M_{0}\right\}}{M_{0}}}|\{\phi, h\}-\{\psi, k\}|_{X} e^{\sqrt{M_{0}} \beta t}\right]$.

Therefore the conclusion follows from (3.11) and (3.12).

Proposition 3.8. For any $t_{0}>0$, there exists $M>0$ such that if $\{\phi, h\},\{\psi, k\} \in \Omega$, then

$$
\left|x_{i}^{\psi, k}-x_{t}^{\phi, h}-u_{i}^{\phi, h, \psi-\phi, k-h}\right|_{X} \leqslant M|\{\psi, k\}-\{\phi, h\}|_{X}^{2}
$$

for all $t \in\left[0, t_{0}\right]$.

Proof: Let

$$
L_{2}:=\sup _{\Phi \in \overline{c o}\left(P_{1} \Omega\right)}\left|D^{2} f(\Phi)\right|
$$


Since $f$ is $C^{2}$ and $\overline{c o}\left(P_{1} \Omega\right)$ is compact, $L_{2}<\infty$. To simplify the presentation, we set $x(t)=x^{\phi, h}(t), y(t)=x^{\psi, k}(t), u(t)=u^{\phi, h, \psi-\phi, k-h}(t)$, and $w(t)=y(t)-x(t)-u(t)$ for $t \geqslant-r$. Applying $P_{2}$ on both sides of (3.6) and using Proposition 3.2 and 3.5, we obtain

$$
u(t)=S(t) u(0)+\int_{0}^{t} S(t-s) D f\left(x_{\diamond}\right) u, d s, \quad t \geqslant 0 .
$$

Therefore from (3.8) and (3.9), it follows that

$$
\begin{aligned}
w(t) & =\int_{0}^{t} S(t-s)\left[f\left(y_{0}\right)-f\left(x_{s}\right)-D f\left(x_{0}\right) u_{s}\right] d s \\
& =\int_{0}^{t} S(t-s)\left[\int_{0}^{1} D f\left(\tau y_{s}+(1-\tau) x_{s}\right)\left(y_{s}-x_{s}\right) d \tau-D f\left(x_{s}\right) u_{s}\right] d s \\
& =\int_{0}^{t} S(t-s)\left[\int_{0}^{1} D f\left(\tau y_{0}+(1-\tau) x_{s}\right) d \tau-D f\left(x_{s}\right)\right]\left(y_{s}-x_{s}\right) d s \\
& +\int_{0}^{t} S(t-s) D f\left(x_{s}\right) w_{s} d s \\
& =\int_{0}^{t} S(t-s)\left[\int _ { 0 } ^ { 1 } \int _ { 0 } ^ { 1 } D ^ { 2 } f \left(\theta\left(\tau y_{s}+(1-\tau) x_{0}\right)\right.\right. \\
& \left.\left.+(1-\theta) x_{s}\right) d \theta \tau\left(y_{s}-x_{s}\right) d \tau\right]\left(y_{s}-x_{s}\right) d s+\int_{0}^{t} T(t-s) D f\left(x_{s}\right) w_{s} d s
\end{aligned}
$$

Since $x_{s}, y_{\varepsilon} \in P_{1} \Omega$ for all $s \geqslant 0, \theta\left(\tau y_{s}+(1-\tau) x_{s}\right)+(1-\theta) x_{0} \in \overline{c o}\left(P_{1} \Omega\right)$, the closed convex hull of $P_{1}(\Omega)$, for all $\theta, \tau \in[0,1]$. Therefore by (3.13) we get

$$
\begin{aligned}
|w(t)|_{H} & \leqslant \int_{0}^{t} e^{\alpha(t-s)} L_{2}\left|y_{s}-x_{s}\right|_{L^{2}}^{2} d s+\int_{0}^{t} e^{\alpha(t-s)} L_{1}\left|w_{s}\right|_{L^{2} d s} \\
& \leqslant t_{0} \max \left\{e^{\alpha t_{0}}, 1\right\} L_{2} \max _{\bullet \in\left[0, t_{0}\right]}\left|y_{\bullet}-x_{s}\right|_{L^{2}}^{2}+\int_{0}^{t} L_{1} \max \left\{e^{\alpha t_{0}}, 1\right\}\left|w_{\triangleleft}\right|_{L^{2}} d s .
\end{aligned}
$$

By Proposition 3.7, we have

$$
|w(t)|_{H} \leqslant M_{1}|\{\psi, k\}-\{\phi, h\}|_{X}^{2}+M_{2} \int_{0}^{t}\left|w_{s}\right|_{L^{2}} d s,
$$

where $M_{1}=t_{0} \max \left\{e^{\alpha t_{0}}, 1\right\} L_{2} L_{1}^{2}$ and $M_{2}=L_{1} \max \left\{e^{\alpha t_{0}}, 1\right\}$. Therefore,

$$
\begin{aligned}
\left|w_{t}\right|_{L^{2}}^{2} & =\int_{-r}^{0}|w(t+\theta)|_{H}^{2} d \theta \\
& \leqslant 2 M_{1}^{2} r|\{\psi, k\}-\{\phi, h\}|_{X}^{4}+2 M_{2}^{2} r\left(\int_{0}^{t}\left|w_{\iota}\right|_{L^{2}} d s\right)^{2}
\end{aligned}
$$


from which it follows that

$$
\left|w_{t}\right|_{L^{2}} \leqslant \sqrt{2 r} M_{1}|\{\psi, k\}-\{\phi, h\}|_{X}^{2}+M_{2} \sqrt{2 r} \int_{0}^{t}\left|w_{\lrcorner}\right|_{L^{2}} d s .
$$

This implies, by the Gronwall inequality, that

$$
\left|w_{t}\right|_{L^{2}} \leqslant \sqrt{2 r} M_{1}|\{\psi, k\}-\{\phi, h\}|_{X}^{2} e^{M_{2} \sqrt{2 r} t}
$$

Substituting (3.15) into (3.14), we get

$$
|w(t)|_{H} \leqslant M_{1}|\{\psi, k\}-\{\phi, h\}|_{X}^{2}+M_{1}|\{\psi, k\}-\{\phi, h\}|_{X}^{2} e^{M_{2} \sqrt{2 r} t}
$$

for all $t \in\left[0, t_{0}\right]$. The conclusion then follows from (3.15) and (3.16).

THEOREM 3.9. Assume that $f$ is $C^{2}$. The semigroup $\{T(t)\}_{t \geqslant 0}$ is uniformly differentiable in $\Omega$.

Proof: For any given $\{\phi, h\} \in \Omega$ and $t \geqslant 0$, define the linear operator $L(t,\{\phi, h\}): X \rightarrow X$ by:

$$
L(t,\{\phi, h\})\{\psi, k\}=U^{\phi, h, \psi, k}(t), \quad \text { for }\{\psi, k\} \in X .
$$

By Proposition 3.8, we have

$$
\begin{aligned}
& \sup _{\{\phi, h\},\{\psi, k\} \in \Omega, 0<\{\{, h\}-\{\psi, k\} \mid x<e} \frac{|T(t)\{\psi, k\}-T(t)\{\phi, h\}-L(t,\{\phi, h\})(\{\psi, k\}-\{\phi, h\})| x}{|\{\psi, k\}-\{\phi, h\}| x} \\
& \rightarrow 0 \text { as } \varepsilon \rightarrow 0 .
\end{aligned}
$$

Therefore, it suffices to show the linear operator $L(t,\{\phi, h\})$ is bounded.

Let

$$
u(t)= \begin{cases}P_{2} U^{\phi, h, \psi, k}(t) & \text { for } t \geqslant 0 \\ \psi(t) & \text { for } \quad-r \leqslant t<0 .\end{cases}
$$

If $\{\psi, k\} \in D(\bar{A})$, by Proposition 3.6, we have

$$
\dot{u}(t)=A u(t)+D f\left(x_{t}\right) u_{t} \quad \text { for } \quad t \geqslant 0,
$$

where $x=x^{\phi, h}$. Thus

$$
u(t)=S(t) u(0)+\int_{0}^{t} S(t-s) D f\left(x_{\iota}\right) u_{\lrcorner} d s .
$$

From this, it follows that

$$
|u(t)|_{H} \leqslant e^{\alpha t}|k|_{H}+\int_{0}^{t} e^{\alpha(t-s)} L_{1}\left|u_{s}\right|_{L^{2}} d s .
$$


Using a similar argument as in the proof of Propositions 3.7 and 3.8, we see that for any $t_{0}>0$, there exists a constant $V>0$ such that

$$
\left|u_{t}\right|_{x} \leqslant V\left|u_{0}\right|_{x}=V|\{\psi, k\}| x \quad \text { for all } \quad t \in\left[0, t_{0}\right]
$$

Since the mild solution of the initial value problem (3.5) depends continuously on initial data and $D(A)$ is dense in $X,(3.17)$ holds for all $\{\psi, k\} \in X$. Therefore $L(t,\{\phi, h\})$ is a bounded and

$$
\sup _{\{\phi, h\} \in \Omega}|L(t,\{\phi, h\})|_{B L(X ; X)} \leqslant V, \quad \text { for all } t \in\left[0, t_{0}\right]
$$

We are now in a position to estimate the Lyapunov numbers and topological dimensions of the global attractor $\Omega$.

Let $\{\phi, h\} \in \Omega,\left\{\psi_{i}, k_{i}\right\} \in D(\bar{A})$ and $U_{i}=U_{i}^{\phi, h, \psi_{i}, k_{i}}, i=1, \ldots, m$. By Proposition 3.4, we have

$$
\frac{d U_{i}(t)}{d t}=\bar{A} U_{i}(t)+\left\{0, D f\left(x_{t}^{\phi, h}\right)\right\} P_{1} U_{i}(t), \quad t \geqslant 0
$$

Therefore, by employing an argument similar to that for (2.40) in Teman [14, Chapter V], we obtain

$$
\frac{1}{2} \frac{d}{d t}\left|U_{1}(t) \wedge \cdots \wedge U_{m}(t)\right|_{\wedge^{m} X}^{2}=\left|U_{1}(t) \wedge \cdots \wedge U_{m}(t)\right|_{\wedge^{m} X}^{2} \operatorname{Tr}\left(G(t) \circ Q_{m}(t)\right)
$$

where

$$
Q_{m}(t)=Q_{m}\left(t, \phi, h, \psi_{1}, k_{1}, \ldots, \psi_{m}, k_{m}\right)
$$

is the orthogonal projection of $X$ onto the space spanned by $U_{1}(t), \ldots, U_{m}(t)$ and $G(t)=G(t, \phi, h): X \rightarrow X$ is defined by

$$
G(t)\{\psi, k\}=\bar{A}\{\psi, k\}+\left\{0, D f\left(x_{t}^{\phi, h}\right) \psi\right\}
$$

Therefore

$$
\begin{aligned}
& \left|U_{1}(t) \wedge \cdots \wedge U_{m}(t)\right|_{\wedge m X} \\
& =\left|U_{1}(0) \wedge \cdots \wedge U_{m}(0)\right|_{\wedge m X} \exp \left(\int_{0}^{t} \operatorname{Tr}\left(G(\tau) \circ Q_{m}(\tau)\right) d \tau\right) \\
& =\left|\left\{\psi_{1}, k_{1}\right\} \wedge \cdots \wedge\left\{\psi_{m}, k_{m}\right\}\right|_{\wedge m} X \exp \left(\int_{0}^{t} \operatorname{Tr}\left(G(\tau) \circ Q_{m}(\tau)\right) d \tau\right)
\end{aligned}
$$


Let

(3.21) $q_{m}(t)=\sup _{\{\phi, h\} \in \Omega,\left\{\psi_{i}, k_{i}\right\} \in D(\bar{A}),\left|\left\{\psi_{i}, k_{i}\right\}\right|_{x} \leqslant 1, i=1, \ldots, m} \frac{1}{t} \int_{0}^{t} \operatorname{Tr}\left(G(\tau) \circ Q_{m}(\tau)\right) d \tau$ and

$$
q_{m}=\limsup _{t \rightarrow \infty} q_{m}(t)
$$

Then we have

$$
\left|U_{1}(t) \wedge \cdots \wedge U_{m}(t)\right|_{\wedge m X} \leqslant\left|\left\{\psi_{1}, k_{1}\right\} \wedge \cdots \wedge\left\{\psi_{m}, k_{m}\right\}\right|_{\wedge^{m} X} \exp \left\{t q_{m}(t)\right\} .
$$

Since the mild solution of the initial value problem (3.5) depends continuously on initial data and $D(\bar{A})$ is dense in $X,(3.23)$ holds for all $\left\{\psi_{i}, k_{i}\right\} \in X, i=1, \ldots, m$. Therefore using the notations in (2.4)-(2.8), we have the following estimation:

$$
\bar{\omega}_{m}(t) \leqslant \exp \left\{t q_{m}(t)\right\},
$$

and

$$
\pi_{m} \leqslant e^{q_{m}}
$$

Therefore

$$
\mu_{1}+\cdots+\mu_{m}=\ln \pi_{m} \leqslant q_{m}
$$

By Theorem 2.3, we obtain our main result:

\section{ThEOREM 3.10. Suppose that}

(i) (H1)-(H3) are satisfied, and

(ii) $f: L^{2}([-r, 0] ; H) \rightarrow H$ is twice continuously differentiable.

If $q_{m}<0$ for some $m$, then the Hausdorff dimension of $\Omega$ is less than or equal to $m$ and the fractal dimension of $\Omega$ is less than or equal to $m \max _{1 \leqslant j \leqslant m-1}\left(1+\left(q_{j}\right)_{+} /\left|q_{m}\right|\right)$.

REMARK: Some smoothness condition on $f$ (for example, (ii) in Theorem 3.10) seems to be necessary in order for $\Omega$ to be of finite dimension (see Yorke [19]).

\section{REFERENCES}

[1] A.V. Babin and M.I. Vishik, 'Attractors of partial differential equations and estimates of their dimension', Russian Math. Surveys 38 (1983), 151-213. 
[2] A.V. Babin and M.I. Vishik, 'Attracteurs maximaux dans les équations aux dérivées partielles', in Séminaire du Collège du France, Editors H. Brézis and J.L. Lions (Pitnam, Boston, 1985).

[3] P. Constantin, C. Foias and R. Teman, 'Attractors representing turbulent flows', in $\mathrm{Mem}$ oirs of AMS, 53, No. 314 (American Mathematical Society, Providence, 1985).

[4] M. Crandrall and T. Liggett, 'Generation of semigroups of nonlinear transformations on general Banach spaces', Amer. J. Math. 93 (1971), 265-298.

[5] H. Federler, Geometric measure theory (Springer-Verlag, Berlin, Heidelberg, New York, 1969).

[6] J.K. Hale, 'Asymptotic behavior of dissipative systems', in Mathematical Surveys and Monographs 25 (American Mathematical Society, Providence, 1988).

[7] T. Kato, 'Non-linear semigroups and evolution equations', J. Math. Soc. Japan 19 (1967), 508-520.

[8] N. Koppell and D. Ruelle, 'Bounds on complexity in reaction-diffusion systems', SIAM J. Appl. Math. 46 (1986), 68-80.

[9] B. Mandelbrot, Fractals: Form, chance and dimension (Freeman, San Francisco, 1977).

[10] J. Mallet-Paret, 'Negatively invariant sets of compact maps and an extension of a theorem of Cartwright', J. Differential Equations 22 (1976), 331-348.

[11] M. Marion, 'Attractors for reaction-diffusion equations: existence and estimate of their dimension', Appl. Anal. 25 (1987), 101-147.

[12] M. Marion, 'Finite-dimensional attractors associated with partially dissipative reaction-diffusion systems', SIAM J. Appl. Math. 20 (1989), 816-844.

[13] A. Pazy, Semigroups of linear operators and applications to partial differential equations (Springer-Verlag, Berlin, Heidelberg, New York, 1983).

[14] R. Teman, Infinite-Dimensional Dynamical Systems in Mechanics and Physics (Springer-Verlag, Berlin, Heidelberg, New York,, 1988).

[15] C.C. Travis and G.F. Webb, 'Existence and Stability for partial functional differential equations', Trans. Amer. Math. Soc. 200 (1974), 395-418.

[16] C.C. Travis and G.F. Webb, 'Existence, stability and compactness in the $\alpha$-norm for partial functional differential equations', Trans. Amer. Math. Soc. 240 (1978), 129-143.

[17] G.F. Webb, 'Functional differential equations and nonlinear semigroups in $L^{p}$-spaces', $J$. Differential Equations 20 (1976), 71-89.

[18] G.F. Webb, 'Asymptotic stability for abstract nonlinear functional differential equations', Proc. Amer. Math. Soc. 54 (1976), 225-230.

[19] J.A. Yorke, 'Non-continuable solutions of differential-delay equations', Proc. Amer. Math. Soc. 21 (1969), 648-652.

Department of Mathematica

University of Alberta

Edmonton Alberta

Canada T6G 2G1
Department of Mathematics

York University

North York Ontario

Canada M3J IP3 